\title{
Andrologie et Squelette
}

\author{
R. PERROT \\ Département de Biologie Humaine Lyon ; Laboratoire d'Anthropologie Anatomique et de \\ Paléopathologie ; Lyon
}

\section{RESUME}

Le squelette humain présente, dans son ensemble, la possibilité de diagnostiquer le sexe de l'individu.

L'auteur, Anthropologue-légiste, Expert près la Cour d'Appel de Lyon, depuis près de 30 ans, présente ici les caractéristiques squelettiques du sexe masculin.

Il insiste également sur une variabilité de ces caractéristiques liée:

- au phénotype racial

- à l'âge

- à l'activité physique

- à l'état de santé

Ces facteurs sont à prendre en compte dans la diagnose sexuelle.

Mots-Clés : Squelette humain - Anthropologie médico-légale - Diagnose du sexe masculin.

\section{INTRODUCTION}

Tout individu est désigné nominativement : Mme X, Mr Y, Mlle Y.

Mr, Mme ou Mlle indiquent donc, sans ambiguité (sauf quelques cas particuliers), pour l'état civil, le sexe administratif, reflet socialisé du sexe biologique.

En tant qu'Anthropologue-Légiste, Expert depuis une trentaine d'années, près la Cour d'Áppel de Lyon, la diagnose sexuelle est (avec la race, l'âge et la stature) l'un des éléments que nous recherchons en priorité sur un cadavre inconnu (souvent réduit à des vestiges squelettiques) afin de l'identifier $[6,7]$.

Il nous a paru intéressant de présenter dans le cadre de cette revue, les grandes lignes permettant de reconnaître un individu masculin à partir de son squelette, en insistant sur notre propre méthodologie [6].

\section{GENERALITES SUR LA DIAGNOSE SEXUELLE}

Le squelette présente, dans son ensemble, la possibilité de diagnostiquer le sexe de l'individu.

Cet examen prend en compte des caractères anatomiques (donc descriptifs) auxquels on peut ajouter des valeurs métriques.

Il nous paraît utile, voire indispensable d'attirer immédiatement l'attention du lecteur, sur les limites de la diagnose sexuelle, auxquelles notre longue pratique, nous a confronté.

L'extrème variabilité d'Homo sapiens, intervient obligatoirement au niveau de l'expression osseuse de son sexe. C'est ainsi que :

- le phénotype racial,

- l'âge,

- l'activité physique,

- l'état de santé

vont retentir de manière non négligeable, sur les caractères squelettiques. 
Une femme sportive, une agricultrice, auront des os nettement plus marqués musculairement que ceux d'un homme sédentaire d'où une possible erreur dans la diagnose sexuelle.

Rappelons à ce sujet l'exemple typique des femmes esquimaudes du début de ce siècle, qui présentaient des mastoïdes (classiquement très développées dans le sexe masculin) plus volumineuses que celles de leurs congénères mâles, du fait qu'elles passaient une bonne partie de leur temps à mastiquer des peaux de phoques!

Sur ce plan pratique nous utilisons une technique dérivée des travaux de deux anthropologues hongrois [1].

Nous avons retenu 44 caractères à valeur sexuelle, répartis de la manière suivante :

\begin{tabular}{llll}
\hline Caractères & Crâne & \multicolumn{2}{c}{ Post-crâne } \\
\hline descriptifs & 7 & 6 & 13 \\
métriques & 2 & 29 & 31 \\
\hline Total & $\mathbf{9}$ & $\mathbf{3 5}$ & $\mathbf{4 4}$ \\
\hline
\end{tabular}

A chaque caractère est attribuée une valeur numérique coefficientée, selon le modèle cijoint :

\begin{tabular}{lcccc}
\hline Caractères & Coef. & -1 & 0 & +1 \\
\hline $\begin{array}{l}\text { Zone glabellaire } \\
\text { Poids crânien }\end{array}$ & 1 & $\begin{array}{c}\text { aplatie } \\
\text { inf.ou }=400 \mathrm{~g}\end{array}$ & $\begin{array}{r}\text { saillante } \\
\text { sup.ou }=800 \mathrm{~g}\end{array}$ \\
\hline
\end{tabular}

Un caractère féminin sera indiqué par -1 , un maculin par +1 , si le caractère est intermédiaire et qu'on ne puisse pas déterminer le sexe on indiquera 0 .

La valeur retenue $(-1,0$ ou +1$)$ est multipliée par le coefficient correspondant au caractère. On fait ensuite la somme totale des coefficients, la somme totale algébrique des valeurs sexuelles, et on divise la seconde par la première, le résultat obtenu, pré- cédé du signe + ou -, indique le sexe à retenir (Tableau 1).

Dans ce qui suit, nous ne retiendrons que les variations masculines du squelette, en traitant sucessivement : crâne, rachis, ceinture scapulaire, ceinture pelvienne, os des membres, extrémités distales.

\section{CRANE}

15 caractères sont pris en compte au niveau du crâne et de la mandibule dont 10 sont purement anatomiques (Tableau 2, Figure 1 et 2).

La lecture de ces deux tableaux amène quelques remarques importantes. On note qu'un caractère coefficienté 2 est, évidemment, plus important pour la diagnose sexuelle que celui coefficienté 1 et doit donc être recherché en priorité.

C'est le cas au niveau du crâne masculin, pour :

- la zone glabellaire, nettement saillante

- le rebord orbitaire supérieur, mousse

- la zone iniaque, saillante,

- les apophyses mastoïdes, massives et pour la mandibule :

- la longueur totale, supérieure ou égale à $112 \mathrm{~mm}$

- la largeur bigoniaque supérieure ou égale à $103 \mathrm{~mm}$

- le poids, supérieur ou égal à $80 \mathrm{~g}$.

Concernant ce dernier paramètre, il faut préciser sa grande variabilité, en ce qui concerne le crâne, liée à un ensemble de facteurs ostéolytiques : masse cérébrale, $\mathrm{pH}$ du sol, lessivage par les eaux de ruissellement, action des organismes vivants (rongeurs, vers de terre, insectes, etc...).

La mandibule, plus massive, résiste davantage d'où le coefficient de 2 attribué à son poids.

Seules les deux premières cervicales sont utilisables. $\mathrm{C}_{1}$ et $\mathrm{C}_{2}$ sont masculines quand leur diamètre transverse maximal est supérieur ou égale respectivement, à 80 et $60 \mathrm{~mm}$. 
Tableau 1 : Exemple théorique d'application de la méthode pour sexer un squelette (ici le sexe féminin pourrait être retenu).

\begin{tabular}{|c|c|c|c|c|}
\hline Caractères & Coef & & Dia & gnose sexuelle \\
\hline $\mathrm{A}$ & 2 & $\mathrm{X}$ & -1 & $=-2$ \\
\hline B & 2 & $\mathrm{X}$ & 0 & $=$ \\
\hline $\mathrm{C}$ & 1 & $\mathrm{X}$ & -1 & $=0$ \\
\hline $\mathrm{D}$ & 2 & $\mathrm{X}$ & 0 & $=0$ \\
\hline $\mathrm{E}$ & 1 & $\mathrm{X}$ & +1 & $=+1$ \\
\hline $\mathrm{F}$ & 1 & $\mathrm{X}$ & 0 & $=$ \\
\hline G & 2 & $\mathrm{X}$ & -1 & $=-2$ \\
\hline $\mathrm{H}$ & 1 & $\mathrm{X}$ & -1 & $=$ \\
\hline I & 2 & $\mathrm{X}$ & 0 & $=0$ \\
\hline$J$ & 2 & $\mathrm{X}$ & +1 & $=+2$ \\
\hline Etc ... & & & & \\
\hline
\end{tabular}

Résultat final : $\underline{\text { Diagnose sexuelle }}=-0,18$ Coefficients
CEINTURE SCAPULAIRE (Figure 3 et 4)

Elle est constituée de la scapula et de la clavicule. En dehors des insertions musculaires, particulièrement le grand Pectoral sur la clavicule, dont l'empreinte forte signe normalement le sexe masculin, 9 paramètres sont retenus, 5 d'entre-eux sont importants (coefficient 2).

Une clavicule sera masculine si :

- sa longueur maximale est supérieure ou égale à $150 \mathrm{~mm}$

- son périmètre au milieu, est supérieur ou égal à $36 \mathrm{~mm}$

- et si l'indice de robustesse, découlant des deux mesures précédentes, est supérieur ou égal à 24 .

Il en sera de même pour la scapula, si la hauteur et la largeur de la cavité glénoïde sont supérieurs ou égales, respectivement à $157 \mathrm{~mm}$ et $29 \mathrm{~mm}$.

Tableau 2 : Caractères sexuels du crâne et de la mandibule.

\begin{tabular}{|c|c|c|c|}
\hline Caractères & Coef. & -1 & +1 \\
\hline Contour général & 1 & Régulier et arrondi & Irrégulier et anguleux \\
\hline Zone glabellaire & 2 & Aplatie & Nettement saillante \\
\hline Rebord orbitaire supérieur & 2 & Aigu et tranchant & Non aigu mais mousse \\
\hline Frontal & 1 & Bombé et arrondi & Aplati \\
\hline Zone iniaque & 2 & Non saillante & Saillante \\
\hline Impressions nuchales & 1 & Nulles à effacées & Très marquées \\
\hline Apophyses mastoïdes & 2 & $\begin{array}{l}\text { Très petites,nettement } \\
\text { AU-DESSUS du plan } \\
\text { des condyles occipitaux }\end{array}$ & $\begin{array}{l}\text { Longues, nettement } \\
\text { AU-DESSOUS du plan } \\
\text { des condyles occipitaux }\end{array}$ \\
\hline Poids crânien & 1 & Inf. ou $=400 \mathrm{~g}$ & Sup. ou $=800 \mathrm{~g}$ \\
\hline $\begin{array}{l}\text { Poids des deux fémurs } \\
\text { comparé au poids du crâne }\end{array}$ & 1 & $\begin{array}{l}\text { Poids des } 2 \text { fémurs } \\
\text { inf. poids du crâne } \\
(=\text { minimum requise }=50 \mathrm{~g})\end{array}$ & $\begin{array}{l}\text { Poids des } 2 \text { fémurs } \\
\text { sup. poids du crâne } \\
(=\text { min. de } 50 \mathrm{~g})\end{array}$ \\
\hline Longueur totale & 2 & Inf. ou $=95$ & Sup. ou $=112$ \\
\hline Largueur bigoniaque & 2 & Inf. ou $=87$ & Sup. ou = 103 \\
\hline Poids & 2 & Inf. ou $=38 \mathrm{~g}$ & Sup. ou $=80 \mathrm{~g}$ \\
\hline Contour général & 1 & Arrondi & Anguleux \\
\hline Menton & 1 & Arrondi et peu saillant & Saillant et carré \\
\hline Gonion & 1 & Non exoversé & Exoversé \\
\hline
\end{tabular}




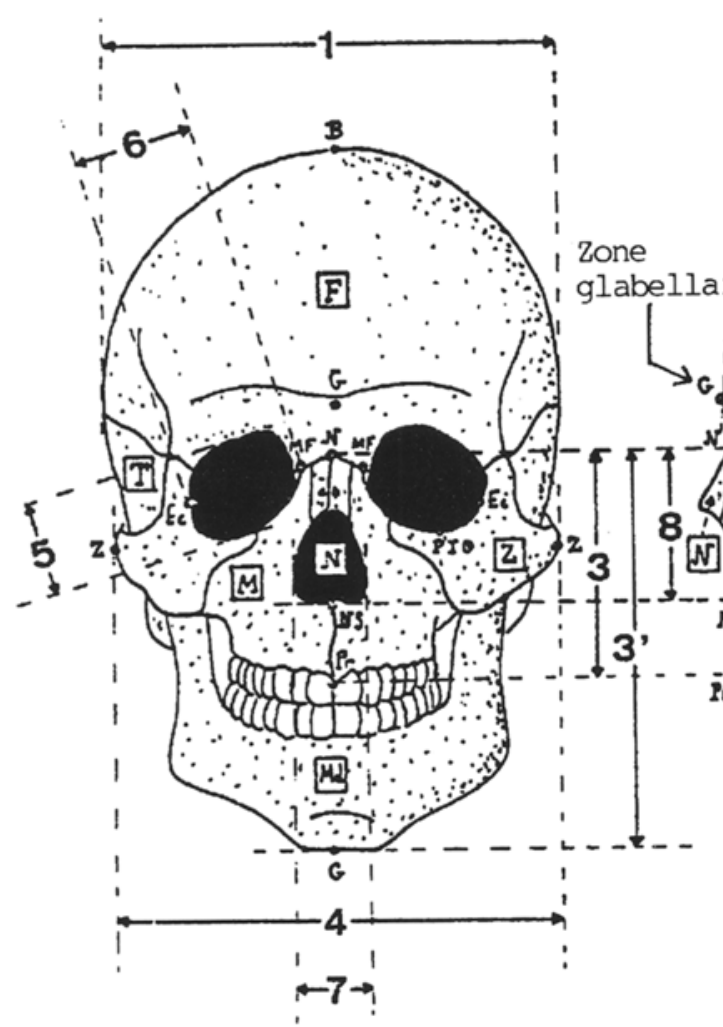

\begin{tabular}{|c|c|c|}
\hline \multicolumn{3}{|c|}{ Points craniométriques } \\
\hline \multirow[t]{4}{*}{ Crâne cérébral } & $\mathrm{B}$ & Bregma \\
\hline & $\mathrm{L}$ & Lambda \\
\hline & $\mathrm{Op}$ & Opisthocranion \\
\hline & Po & Porion \\
\hline \multirow[t]{8}{*}{ Crâne facial } & Ec & Ectoconchion \\
\hline & $\mathrm{G}$ & Glabelle \\
\hline & $\mathrm{MF}^{\mathrm{H}}$ & Point maxillo-frontal \\
\hline & $\mathrm{N}$ & Nasion \\
\hline & NS & Point nasospinal \\
\hline & $\mathrm{PIO}$ & Point infra-orbitaire \\
\hline & $\operatorname{Pr}$ & Prosthion \\
\hline & $\mathrm{Z}$ & Zygion \\
\hline
\end{tabular}

Mandibule G Gnathion
Morphologie crânienne (os)

$\begin{array}{ll}\mathrm{F} & \text { Frontal } \\ \mathrm{M} & \text { Maxillaire } \\ \mathrm{Md} & \text { Mandibule } \\ \mathrm{N} & \text { Nasale } \\ \mathrm{Oc} & \text { Occipital } \\ \mathrm{P} & \text { Pariétal } \\ \mathrm{S} & \text { Sphénoïde } \\ \mathrm{T} & \text { Temporal } \\ \mathrm{Z} & \text { Zygomatique }\end{array}$

\section{Orientation du crâne}

Selon le plan de Francfort P.F.

\section{Mensuration}

Crâne : 1 largeur, 2 longueur A.P. (indice crânien : 1/2)

Face: 3 hauteur sup., 3' hauteur totale 4 largeur (indice facial : $3 / 4-3{ }^{\prime} f$ )

Orbite : 5 hauteur, 6 largeur (indice orbitaire : $5 / 6$ )

Nez : 7 largeur, 8 hauteur (indice nasal : 7/8)

\section{Figure 1 : Grandes lignes de l'anthropologie cranienne.}



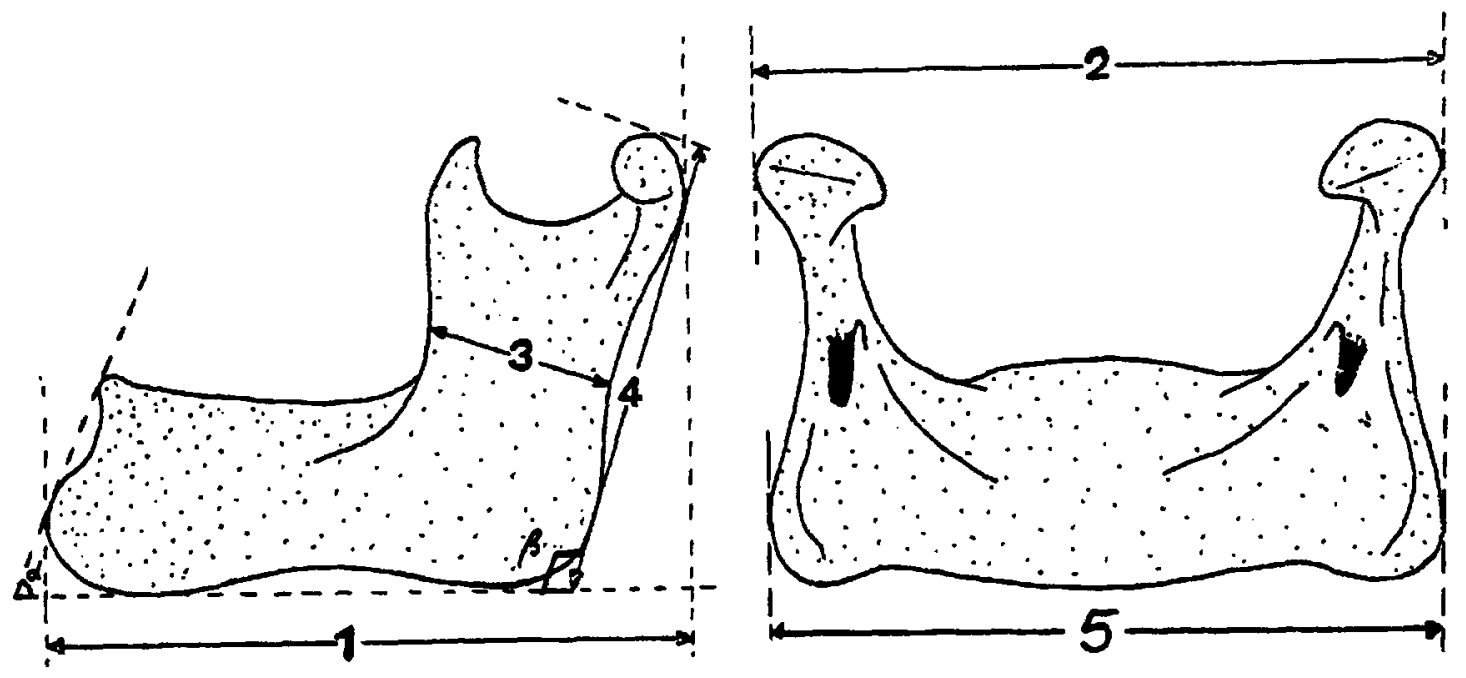

Figure 2 : Principales mensurations mandibulaires

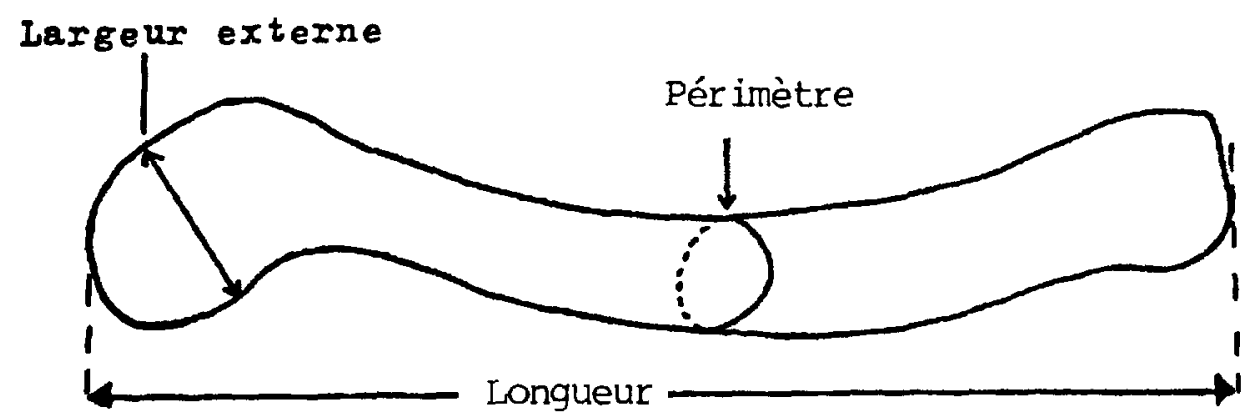

Figure 3 : Clavicule droite (anthropométrie)

\section{LA CEINTURE PELVIENNE (Figure 5)}

Le bassin osseux en particulier l'os coxal est classiquement considéré comme primordial dans la diagnose sexuelle, même au niveau de fragments isolés. Dans la pratique quotidienne, celà est moins évident et justifie les nombreux travaux consacrés à la sexualisation du coxal [1-5].

La plupart du temps, en effet, le bassin osseux présente un panachage de caractères anatomiques mâles et femelles et la diagnose finale va dépendre finalement des éléments majoritairement exprimés dans un sens ou dans l'autre. De ce fait, on comprend aisément que la fiabilité ne puisse jamais atteindre $100 \%$.

En ce qui nous concerne, nous retenons comme caractères descriptifs masculins :

- l'aile iliaque, épaisse et opaque

- le trou obturateur, ovalaire à rebord interne mousse

- la grande échancrure sciatique, très fermée en forme de $\mathrm{V}$

- la branche ischio-pubienne, convexe. On peut prendre en compte également 4 paramètres : 


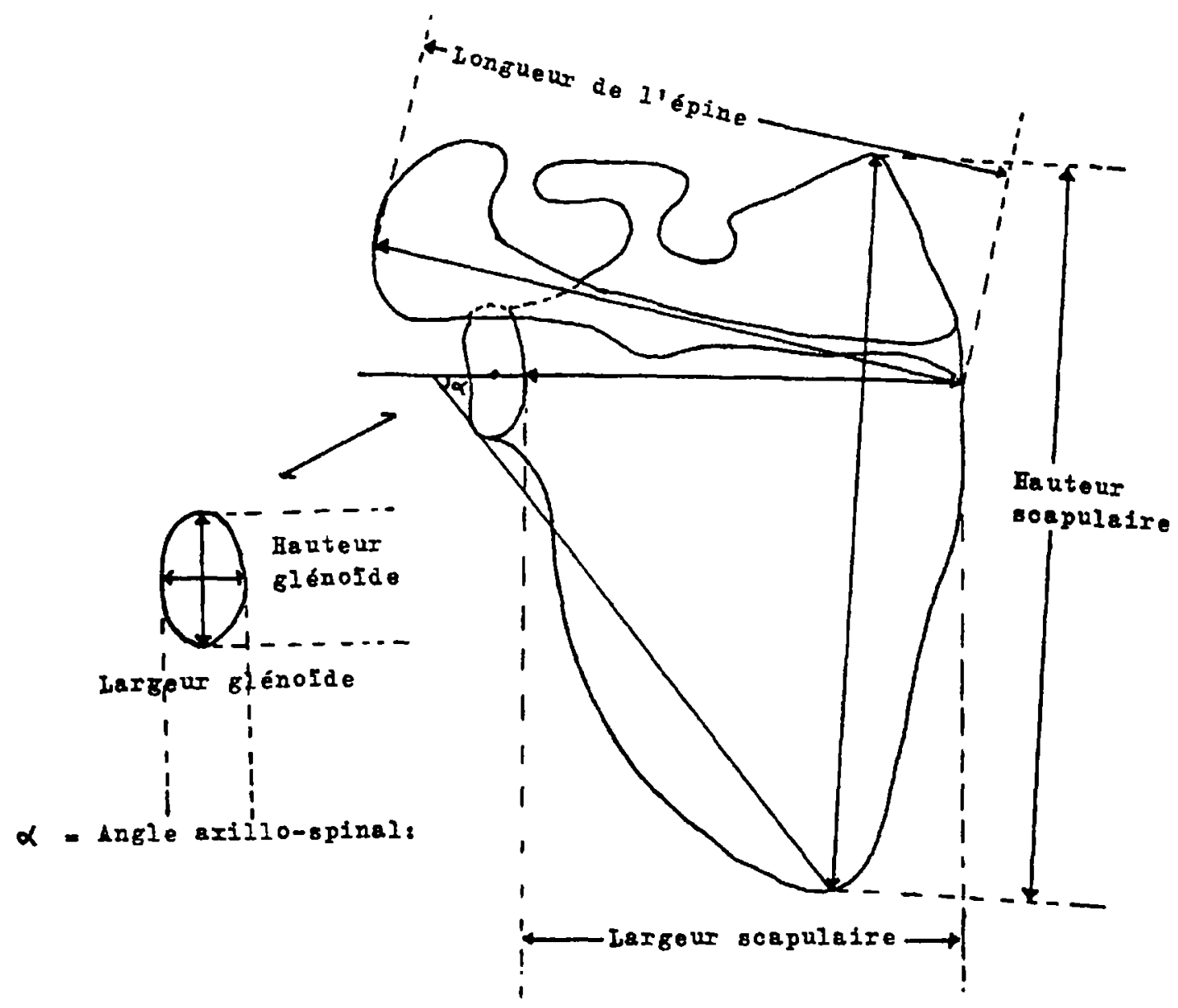

Figure 4 : Scapula gauche (anthropométrie)

- l'indice ischio-pubien masculin pour une valeur inférieure ou égale à $50 \mathrm{~mm}$

- la largeur cotylo-sciatique, supérieure à $40 \mathrm{~mm}$

- l'ouverture de la grande échancrure sciatique, inférieure ou égale à $41 \mathrm{~mm}$.

Les deux paramètres précédents, associés, donnent l'indice cotylo-sciatique, dont les valeurs masculines évoluent entre 83 et 127.

\section{OS DES MEMBRES}

(Figure 6)

Concernant les os longs c'est en priorité la longeur maximale (humérus, ulna, radius, tibia) ou physiologique (fémur) qui est rete- nue. Pour ce dernier os, en particulier, le plus caractéristique de l'espèce humaine dans son adaptation à la bipédie, une longueur supérieure ou égale à $460 \mathrm{~mm}$, signe son appartenance masculine, sexe confirmé si on peut remarquer une ligne âpre (fortes insertions musculaires) marquée à très marquée, et un indice pilastrique (qui lui est correllé) dont la valeur est supérieure ou égale à 115 .

\section{EXTREMITES DISTALES}

L'examen des os des mains et des pieds n'est pas d'un usage courant dans la diagnose sexuelle. Cependant un talus ou un calcaneum particulièrement volumineux indiquent sans difficultés le sexe masculin. 


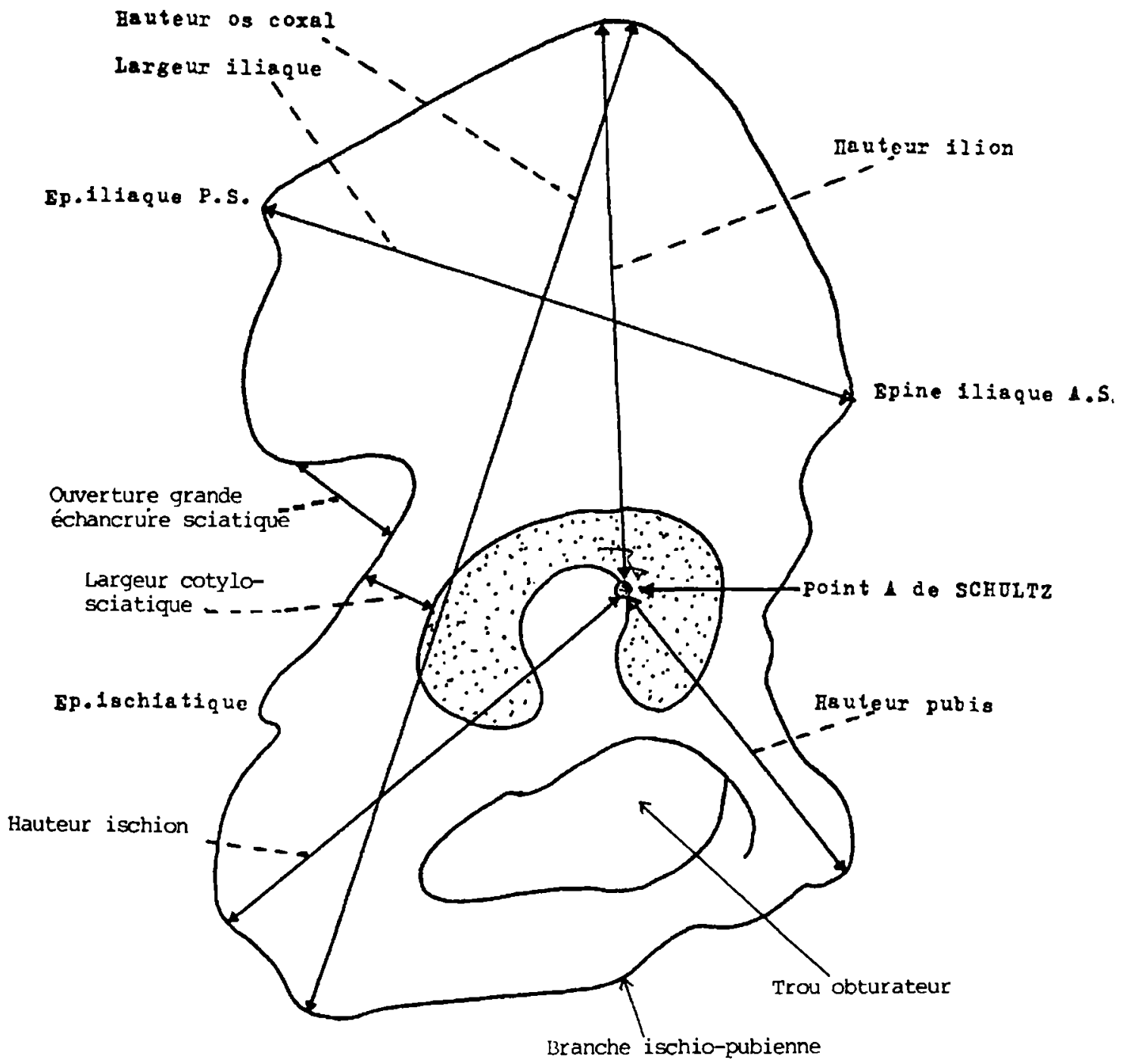

Figure 5 : Principaux paramètres d'un os coxal droit

\section{CONCLUSION}

La diagnose du sexe masculin à partir du squelette fait intervenir un ensemble de caractères anatomiques et métriques. Cependant comme le font remarquer ASCADI et NEMESKERI (avis que nous partageons pleinement) : "Il est souvent possible de distinguer des hommes masculins, hypermasculins et même fémino-masculins ! Dans certains cas les caractères sexuels sont flous et le sujet est indifférencié, ce qui pose des problèmes non négligeables : la diagnose sexuelle devient alors très problématique" [1].
Dans la pratique, l'idéal est de posséder la totalité du squelette, auquel cas, la probabilité d'erreur dans la sexualisation ne dépasse pas $2 \%$ et peut même être nulle quand lindividu est bien "typé" morphologiquement.

Il est intéressant de noter que le pourcentage d'erreur varie en fonction des os, selon qu'ils sont pris isolément ou associés avec d'autres : les valeurs que nous donnons ci-après le sont à titre indicatif, elles représentent l'ordre de grandeur moyen de nos propres estimations et de celles les plus classiquement relevées dans la littérature anthropologique. D'une manière générale la probabilité varie de 2 à $20 \%$. 


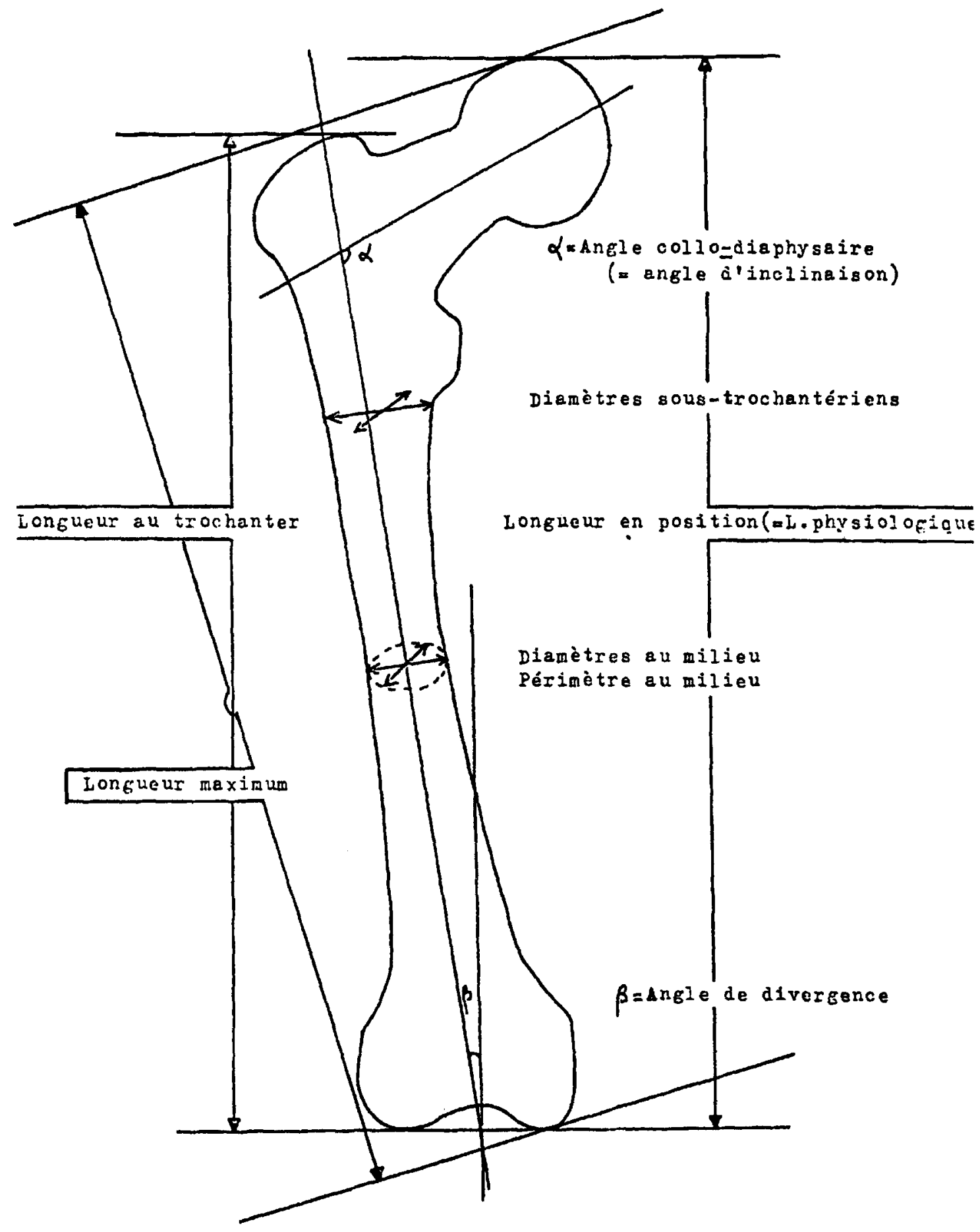

Figure 6 : Anthropométrie du fémur gauche 
La valeur la plus faible correspond à deux ensembles possibles : crâne - mandibule et bassin ou os longs et bassin.

Le bassin seul entraîne 5\% d'erreurs.

Crâne et mandibule pris isolément peuvent être responsables de 9 à $10 \%$ de confusion. Les os longs, non associés au bassin ,fournissent de 15 à $20 \%$ d'erreurs.

Et c'est le crâne sans sa mandibule qui peut entraîner une sexualisation fausse dans $20 \%$ des cas!

Dans les cas litigieux (fréquents en anthropologie historique et surtout en médecinelégale, où la connaissance du sexe est un impératif, on retient, dans les faits, le sexe dont les caractéristiques sont le plus exprimées.

\section{REFERENCES}

1. ASCADI GY, NEMESKERI J. : History of human life span and mortality. Akademiai Kiado, Budapest , 1970, 346p.

2. BRUZEK J. : Fiabilité des procédés de détermination du sexe à partir de l'os coxal. Implications à l'étude du dimorphisme sexuel de l'Homme fossile. Thèse Dr., Muséum Nat. Hist. Nat., Institut de Paléontologie Humaine, Paris, : 1991, 431p. et 102 p. d'annexes.

3. GENOVES S. : L'estimation des différences sexuelles dans l'os coxal. Bull. Soc. Anthrop. Paris, 1959, 10, x,1, 3-95.
4. NOVOTNY V. : Pohlavni rozdily a identifikace pohlavi panevni kosti Sex differences and identification of sex in pelvic bone). $\mathrm{Ph} \mathrm{D}$. Thesis, University J.E. Purkyne, Brno, 1981, 233 p. et annexes.

5. OLIVIER G. : Pratique anthropologique. Vigot, Paris, 1960, 295p.

6. PERROT R. : Documents d'Anthropologie et de Paléopathologie. Univ. Lyon I, Dép. Biol. Humaine, 1977-1978-1980.

7. PERROT R, DAVID JJ. : Les problèmes d'identification en médecine légale, l'apport de l'anthropologie. Méd. Lég. et dommage corps, 1974, VI, 4, 392-396.

\section{ABSTRACT \\ Andrology and skeleton}

R. Perrot

The human skeleton offers the possibility of sex diagnosis.

The Author, forensic anthropologist near the court of Lyon, for about 30 years, presents the male bone features.

He also lays stress on the variability of these features according to : racial phenotyp, age, physical activity and health.

These features are to be considered when sexing human skeleton.

Key-words : Human skeleton - Forensic anthropology - Male sex diagnosis. 